groups. In these respects, the design of many of the studies included in this review was weak and it may be argued that such studies, when giving negative results, provide little evidence to substantiate the product's safety.

Postmarketing surveillance is an evolving subject in which there are several logistic difficulties in designing and performing satisfactory studies. Computerised record linkage schemes need to be expanded to provide a data resource for studies after marketing. In the meantime, a strong case can be made for attempting to improve company sponsored postmarketing surveillance by using the experience of the past four years.

We suggest that three approaches to achieving this objective require consideration. Firstly, a variety of study designs need to be used to study drug safety, and the design of observational cohort studies requires refinement to avoid the problems we have described.
Secondly, we believe that postmarketing studies should place greater emphasis on specific potential safety issues, identified from preclinical studies, premarketing trials, or other postmarketing experience (for example, spontaneous adverse reaction reporting schemes). Finally, logistic problems in recruitment of patients and adequate provision of information to regulatory authorities need to be resolved. To achieve these goals the quadripartite guidelines require revision and discussions on them are underway.

1 Rawlins MD, Jefferys DB. Study of United Kingdom product licence applications containing new active substances, 1987-9. BMf 1991;302:223-5. 2 Committee on Safety of Medicines Second Working Party on Adverse Reactions. Recommendations. In Mann RD, ed. Adverse Drug Reaction Carnforth: Parthenon, 1987:62-3.

3 Joint Committee of ABPI, BMA, CSM, and RCGP. Guidelines on postmarketing surveillance. $B M \mathcal{F} 1988 ; 296: 399-400$.

(Accepted 23 March 1992)

\title{
Effective audit: reporting to the National Confidential Enquiry into Perioperative Deaths
}

\author{
L Clark, P Doyle, E Duran, P Kishore
}

\begin{abstract}
Objective-To investigate the effectiveness of computer based and manual district and unit information systems for identifying hospital deaths eligible for reporting to the National Confidential Enquiry into Perioperative Deaths (NCEPOD).

Design-Prospective six to 10 week study of hospital (death register, immediate coding of medical records) and district information systems followed by six month assessment after modification to entry of data.
\end{abstract}

Setting-Acute units within Lewisham and North Southwark Health District.

Patients-All 290 patients dying in hospital during the six weeks, for whom the medical records were obtainable in 231.

Main outcome measures-Sensitivity and specificity of the information systems in ascertaining eligible surgical deaths (patients dying in hospital who had during 30 days previously had a surgical procedure while under the care of a consultant in a surgical specialty) tested against validated list of screened medical records.

Results-Of 231 medical records, 30 (12 from Lewisham, 18 from North Southwark) met the national inquiry's criteria. The computer based systems of both units detected less than $60 \%$ of eligible deaths (sensitivity $53 \%$, specificity $83 \%$ ); the death register detected about $60 \%$ (sensitivity $61 \%$, specificity $89 \%$ ); manual systems detected all eligible deaths. Subsequent modification to ensure immediate coding of records into the computerised systems during follow up failed to show any improvement.

Implications-Routine hospital information systems may miss up to half the deaths eligible for NCEPOD.

\section{Introduction}

A system of surgical audit-the National Confidential Enquiry into Perioperative Deaths (NCEPOD) -was introduced throughout the United Kingdom on 1 January 1989. The report of the original Confidential Enquiry into Perioperative Deaths emphasised that the validity of clinical audit systems depends on the inclusion of all relevant cases. ${ }^{1}$ Consultants were therefore appointed as local district reporters to notify to the NCEPOD office certain details (name; date of birth; sex; hospital number; date of operation; date of death; consultant in charge; and anaesthetist, if known) of all patients under the care of a consultant in a surgical specialty who died in hospital within 30 days of having undergone a surgical procedure. The notional inquiry would later obtain additional details abo it the perioperative care of such patients from the participating surgeons and anaesthetists for further analysis. ${ }^{2}$ The success of the national inquiry depends on the ability of the local reporters to identify and report all eligible deaths.

To assess this ability we carried out a study in a large inner London teaching district with two units, Guy's Hospital and Lewisham (Lewisham, Sydenham Children's, and Hither Green Hospitals). We examined the routine information systems and assessed their effectiveness in terms of the ascertainment of cases and the provision of the eight data items required by the local reporter for the national inquiry. Methods identified would need to maintain confidentiality and be sensitive (identifying all eligible deaths), specific (eliminating false positives), and robust over time.

\section{Methods}

There was no ward based computerised patient record system in the two units examined, although a ward financial information project was planned. The only comprehensive source of patient data at the time of death was the patient's written medical records. After death or discharge coding clerks in both units entered the data into a computer based patient administration system (ICL Dual 2966 based system, designed by the Inter-Regional Consortium). There was an important difference between the two units. In Guy's Hospital the medical records of patients discharged or dying in hospital were normally released before coding for discharge letters to be written, etc, only being returned for coding after an interval (sometimes weeks). At Lewisham the notes were normally not released until at least the initial coding was complete. In both units there was a backlog of
Beeches, 20 Rectory Close, GU4 7AR 
coded data awaiting entry on to the patient information system. Data on patients dying in hospital could be obtained from several other sources (box). Each potential source of patient information was evaluated for its ability to identify eligible deaths and to provide the eight data items needed correctly. None was capable of providing all eight data items routinely. Three systems seemed to have the potential to identify at least the eligible deaths.

If coding and data entry are timely and complete then the district information system based on the patient information system should be able to identify eligible deaths for both units. The reporting system (Querymaster) for the district information system was therefore programmed to identify all "surgical deaths" - that is, patients dying in hospital within 30 days of an episode of care by a consultant surgeon. At Guy's details of deceased patients were entered into a death register before the medical records were released to the coroner, mortuary, or medical secretaries. A reporter could identify "surgical deaths" from entries in the death register and cross check with the medical records (or theatre financial information project) to confirm that an operation had taken place within the past 30 days. At Lewisham, because medical records of patients dying in hospital were coded immediately, coding clerks were asked to identify eligible deaths. To overcome the backlog in both units coding clerks were also asked to enter on to the patient information system data on patients dying in hospital in preference to that on discharged patients.

For six weeks from 1 January 1989, we obtained a weekly list of probable eligible deaths occurring in the preceding seven days from the three systems: the district information system (which covered both acute units), the death register at Guy's, and the coding clerks at Lewisham. We screened the medical records of every patient dying during the study period in the two units to identify all true eligible deaths. This validated list was used to test the sensitivity and specificity of the three sets of test lists. Lists compiled from the district information system were collected for a further four weeks to allow for delays in entry of data.

\section{Results}

The table summarises the results. During the six weeks 290 patients died. Despite our efforts the medical records of 59 patients were not retrievable, and we examined the remaining 231 sets of records (93 from Guy's, 138 from Lewisham), 30 of which (18 from Guy's, 12 from Lewisham) met the national inquiry's criteria. The coding clerks at Lewisham proved to be $100 \%$ accurate, identifying all 12 eligible deaths out of the 138 medical records screened and

Performance of district information system, death register, and coding clerks in identifying deaths eligible for NCEPOD

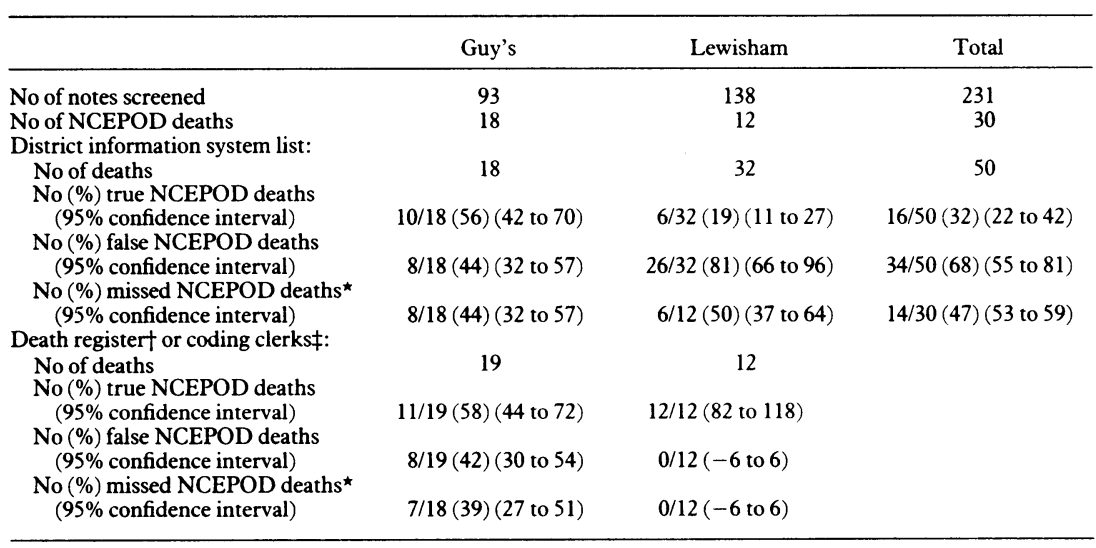

${ }^{\star}$ Missed NCEPOD deaths $=$ NCEPOD deaths - true NCEPOD deaths/NCEPOD deaths. †Guy's. $\ddagger$ Lewisham.

\section{Hospital information systems}

District wide

District information system

Guy's Unit

Theatre financial information project

Patient administration system

Patient registration system (old computer system being phased out)

Death register

Mortuary register

Medical records

Lewisham Unit

Patient administration system

Medical records

providing the eight data items required. The weekly $\vec{\circ}$ district information system list produced a total of 50 . surgical deaths, only 16 of which proved to be true eligible deaths. Fourteen eligible deaths were missed altogether $(47 \%, 95 \%$ confidence interval $35 \%$ to $59 \%)$. 3 The district information system list had a sensitivity of only $53 \%$ and a specificity of $83 \%$. The list from the death register produced 19 surgical deaths, 11 of which were true eligible deaths, but missed seven eligible deaths $(39 \%, 27 \%$ to $51 \%)$, a sensitivity of $61 \%$ and specificity of $89 \%$. None of the lists identified possible eligible deaths among the 59 missing records. In $N$ summary, routine information systems identified at best only $58 \%$ of eligible deaths with significant 5 numbers of false positive results; in contrast, the performance of the Lewisham coding clerks showed $\overparen{D}$ that it is possible to identify accurately and obtain $\vec{e}$ complete information on eligible deaths.

\section{Discussion}

It is neither practicable nor desirable for the local reporters to screen the medical records of every patient dying in hospital even if such records could all be located; reporters should be able to rely on the hospital o information systems. This study suggests that such reliance could be misplaced unless the systems are carefully checked and monitored. False positive cases in small numbers are not important because they can normally be eliminated without difficulty and will not affect the validity of the national inquiry. However, if $\dot{0}$ the findings of this study are representative of those of many other hospitals almost half of the eligible deaths $\delta$ are likely to be missed. Underreporting on this scale will have serious implications for the accuracy and 윽 credibility of the national inquiry.

As a result of this study changes were made to facilitate local reporting. To capitalise on the success of $\frac{}{0}$ the coding clerks the clerks in both units were asked to code the records of all patients dying in hospital immediately after death and to enter the details $\omega$ preferentially into the patient information system. They were also asked to complete a "return" (based on 0 the NCEPOD form) for every death meeting the $\mathbb{D}$ national inquiry's criteria and to send it to the local ? reporter. The reporter would then check the details before formally reporting the death to NCEPOD. As $\frac{0}{\mathbb{D}}$ all the relevant data should also have been entered immediately into the patient information system the $\stackrel{\mathcal{D}}{\circ}$ district information system should be able to provide? the same information. That reporting system was 8 reprogrammed to produce a monthly list of eligible? deaths with all the relevant details. The intention waso to check the computer generated data against the? returns from the coding clerks for several months. If the lists corresponded the district information system would provide a single districtwide computer based reporting system for the national inquiry. 
The returns and computer district information system lists were checked by us after six months. It was clear that, although the average rate of surgical deaths had continued its previous trend, the number of eligible returns was very variable. The returns from one unit were as expected for the first three months and then tailed off rapidly. The only returns from the other unit were for two months in the middle of the six month period. The computer lists were equally poor, identifying only about half of the eligible deaths notified in the returns. The results of the follow up were disappointing and illustrate the problems inherent in implementing and maintaining an accurate audit system. Manually compiled audit systems depend on the knowledge, understanding, and motivation of the staff involved. In this instance, coding clerks have the appropriate skill and can undoubtedly perform well if they understand the problem and receive the medical records immediately after death. However, interest and performance in any task is likely to diminish with time so that a manual system of the type set up in this district will continue to work only if there is regular feedback and monitoring.

\section{RELIABILITY OF COMPUTER BASED SYSTEMS}

In theory computer systems should be reliable. However, systems like the patient information system or district information system in this district depend on complete and accurate coding by the same clerks who complete the NCEPOD returns and are therefore subject to the same problems. Even if all the required data are entered and the patient information system or district information system record contained a large number of data fields, few of them (other than patient name, number, consultant, etc) could be used to generate lists for audit. It proved difficult to pro- gramme the system to generate a list based on, and containing, the data items required by NCEPOD. Newer ward based computer systems being installed as part of resource management initiatives may have advantages. Data on patients will be entered sequentially, much of it by clinical staff, so that as the patient's stay progresses all the relevant data items (date of procedure, surgeon, etc) will be added.

With such systems it should be possible automatically to check the records of any patient dying in hospital for eligibility for the national inquiry and to generate a complete report. Some of the newer systems can also code automatically (for example, for Office of Population Censuses and Surveys, ICD, and Read classifications). Until such systems are widely available, however, timely and accurate coding and entry of all relevant data by well trained and motivated staff remain the key to successful audit.

Provider units are advised to check current methods of reporting to NCEPOD. Managers and clinicians are also advised to check the specification of proposed computer based clinical or patient information systems to ensure they have adequate fields for clinical data and that these can be used to generate reports suitable for the national inquiry or any other audit process.

We thank Dr Sue Atkinson; consultant staff in the surgical specialties, anaesthetics, and pathology; the information and records staff in Lewisham and North Southwark district and Peter Drury, London School of Hygiene and Tropical Medicine, for their help.

1 Buck N Devlin HB, Lunn JN Report of a confidential enguiry into perioperative deaths London: Nuffield Provincial Hospitals Trust, King's Fund, 1987. 2 Protocol for the National Confidential Enquiry into Perioperative Deaths. London National Confidential Enquiry into Perioperative Deaths, 1988

(Accepted 10 April 1992)

\title{
Value of routine ultrasound scanning at 19 weeks: a four year study of 8849 deliveries
}

\author{
Carole A Luck
}

\begin{abstract}
Objective-To evaluate the effectiveness of routine ultrasound scanning at 19 weeks' gestation in an unselected population in terms of accuracy of detection of fetal structural abnormality and the effect on obstetric and neonatal care.

Design-Prospective study over four years. Scans performed by radiographers with overall supervision by a radiologist.

Setting-Ultrasound department of district general hospital.

Subjects - All pregnant women were offered scans; 8523 of $8849(96 \%)$ accepted.

Main outcome measures-Information obtained from hospital records, genetic analysis, and postmortem findings.

Results-166 fetal anomalies occurred; 140 were detected at 19 weeks (sensitivity $85 \%$; specificity $\mathbf{9 9 . 9 \% ) . ~ I n ~} 27$ cases fetuses were shown to have severely crippling or lethal abnormalities; termination of pregnancy was requested in 25 . Early diagnosis influenced timing and place of delivery in babies with severe cardiac or gastrointestinal anomalies.

Conclusion-Scanning at 19 weeks with availability of termination can reduce perinatal morbidity and mortality. Scanning can be performed in a general ultrasound department with adequate counselling
\end{abstract} Ascot, Berkshire SL58A Carole A Luck, consultant radiologist facilities and close cooperation between radiographers, midwives, obstetricians, paediatricians, and the radiologist.

\section{Introduction}

The value of obstetric scanning at 19 weeks' gestation has been assessed in major tertiary referral centres, ${ }^{12}$ but a prospective study that includes centres which are not tertiary referral centres is needed. ${ }^{3}$ Only one study on an unselected population has been recorded, and this emphasised the need for more data to be collected. ${ }^{4}$ The purpose of the present study is to redress this balance and assess the value of 19 week ultrasound scanning in such a population.

A report of the Royal College of Physicians states that routine scanning is offered to pregnant women at only a few centres; in most areas it is available only to women in recognised risk groups. The report continues: "there is good evidence that, with an appropriately organised obstetric ultrasound service, most structural malformations could be detected."

Most analyses agree that screening and prenatal diagnosis programmes are wanted by the population and offer major medical and financial advantages. ${ }^{5}$ The investment required is modest and will conserve NHS resources. Studies show that where risks exist, 